\title{
Smart Patch Antenna for Indoor Mobile Wireless Computing
}

\author{
Min Shi, Junwei Lu, and David J. Ireland \\ Centre of Wireless Monitoring and Applications, Faculty of Engineering and Information Technology, \\ Griffith University, Brisbane, QLD 4111, Australia
}

\begin{abstract}
Two types of circular-polarized smart patch antenna arrays, namely patch antenna with seven hexagonal elements (PASHE) and patch antenna with seven circular elements (PASCE) for indoor mobile wireless computing are investigated. A comparison between simulated and measured reflection coefficient was made with consistent results. The Finite element method (FEM) and optimization using a gradient algorithm was carried out to optimize these patch antennas' physical structures on a finite ground plane to achieve optimal directivity in elevation plane and antenna gain at $2.4 \mathrm{GHz}$ operating frequency. Switched parasitic technique enables the two patch antennas to steer a directional beam through six locations in azimuth with elevation of approximately $65^{\circ}$ for the former and $70^{\circ}$ for the latter respectively. The low profile and low cost characteristics along with the simplistic beam control system of these smart patch antennas are ideal prototypes for commercialization.
\end{abstract}

\section{I . INTRODUCTION}

Smart antenna technology is becoming one of the most effective utilities for improving performance and available spectrum usage demanded by the rapid growing market of wireless communications. Since the early study on reactively controlled directive arrays reported by Harrington [1] attracted many researchers, the research and application of these antenna arrays in mobile wireless communications and computing systems have been carried out and various antenna configurations based on the concept of reactively controlled directive arrays have been reported recently. Among them, Schlub's seven-element ground skirt monopole antenna array based on electronically steerable passive array radiator (ESPAR) for wireless ad hoc computer networks [2] and Lu's dielectric embedded ESPAR antenna array for mobile terminals [3] at $2.4 \mathrm{GHz}$ operating frequency have been successfully developed for mobile wireless communications. Recently, Gray's electronically steerable Yagi-Uda microstrip patch antenna array using switched beam control system for land-mobile communications [4] and Thiel's beam-switched monopole arrays [5] have also been explored for the application in wireless computing and communications systems. However, the antenna array as an access point mounted in the ceiling and facing down to computer users for indoor mobile wireless computing has yet to be reported.

This paper presents two newly developed electronically beam steerable and adaptive microstrip patch antennas, which can be mounted in ceilings for indoor mobile wireless network applications, using either switched parasitic control or reactively loaded method, namely smart patch antenna array with seven hexagonal elements (PASHE) and with seven circular elements (PASCE). These patch antenna arrays have many advantages such as low profile, small size, low cost, ease of mount, low battery consumption. These antenna arrays with adaptive beam-forming circuit can provide ideal communication performance in the wireless local area network (WLAN). Since the Bluetooth and IEEE802.11b or $11 \mathrm{~g}$ standards have become very popular and widely used in wireless computing systems, the wireless security issue becomes the major concern. Unlike traditional antennas, such as omni-directional and directional antennas applied to wireless computing systems, the proposed smart patch antennas can increase the wireless security level in the WLAN system at $2.4 \mathrm{GHz}$ operating frequency by adopting switched beam and adaptive beam-forming technologies to provide adaptive beams to the expected orientations. Furthermore, with the circular-polarized characteristic, these smart patch antennas can sense the signals from indoor mobile wireless terminals in any direction and thus can swiftly find objects. Figure 1 shows the typical application of these smart patch antennas mounted in the ceiling as an access point for indoor WLAN application. These particular antennas can switch beam evenly at six directions in azimuth plane with sufficient signal strength and coverage thus can track all of the indoor mobile wireless terminals.

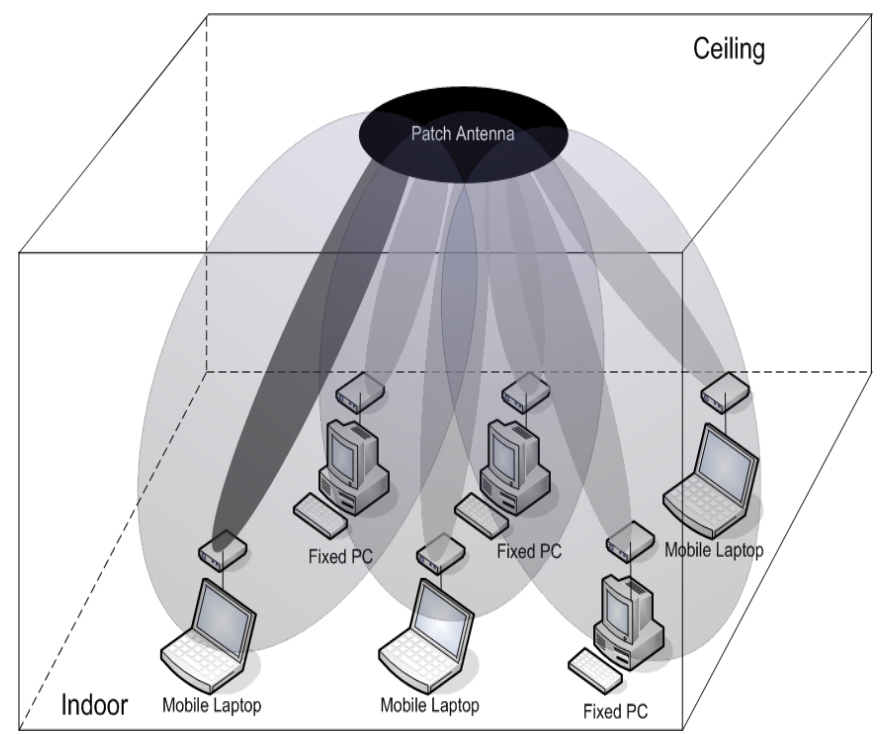

Fig.1 Application for indoor WLAN system by using smart patch antenna 


\section{II . Antenna Design AND Configurations}

Both smart patch antennas have similar configurations, consisting of a single active element surrounded by six equal size parasitic elements situated on top of a printed circuit board (PCB) and with a ground plane located on the bottom side of the PCB forming the well known parallel topology. Figure 2 (a) and (b) shows the configuration of PASHE and PASCE with their respective optimal sizes. Both patch antennas have similar sizes of the central element by approximately $0.15 \lambda$ for PASHE and $0.14 \lambda$ for PASCE respectively. The equal-sized parasitic element of each patch antenna is slightly smaller in size compared to the central element. The size difference between the parasitic element and the central element is $5 \%$ for PASHE and $0.1 \%$ for PASCE respectively. The gap between the central element and the parasitic element is approximately $1 \mathrm{~mm}$ for each of the two patch antennas. The patch array is designed on the FR-4 epoxy/fiber-glass PCB substrate with a thickness $1.6 \mathrm{~mm}$ and dielectric constant 4.4. Both of the smart patch antennas have similar PCB dimensions by approximately $1.02 \lambda$ for PASHE and $0.96 \lambda$ for PASCE respectively.

The central element is fed by two orthogonal feed points with a $90^{\circ}$ phase difference to generate circular polarization of the current distribution. Thus the beam-steering can be carried out equally through six directions in azimuth. Beamsteering is achieved by either open-circuiting or shortcircuiting the parasitic antenna elements by switches or reactive loads where an open-circuited element acts as a dire-

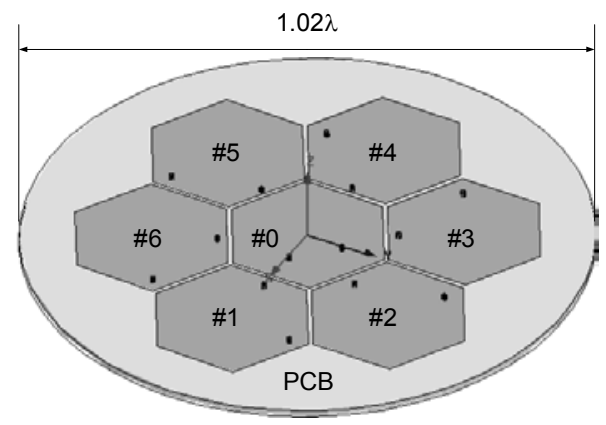

(a)

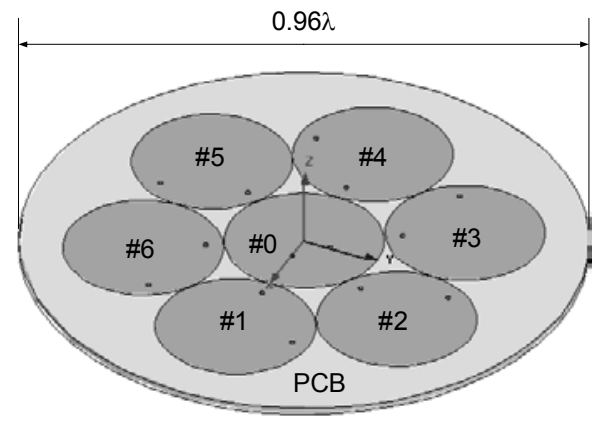

(b)

Fig.2 Configuration of the two smart patch antennas: (a) PASHE; (b) PASCE ctor while a short-circuited element effectively becomes transparent. The fields generated by each element couple together to formulate the total far field. Due to the induced circular-polarized currents in the parasitic elements, two short-circuited points are required for each element to effectively terminate any current presence. The beam orientation and coverage can be controlled by varying either switches or reactive loads in each element to achieve different compositions of open-circuiting and short-circuiting of array elements. A typical beam-forming circuit using switches and the aerial beam-forming structure are illustrated in Fig. 3 (a) and (b) respectively for PASHE and PASCE.

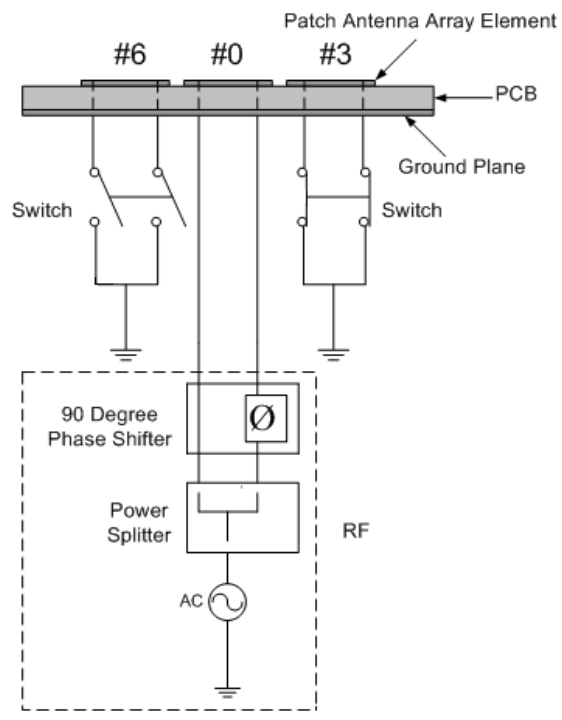

(a)

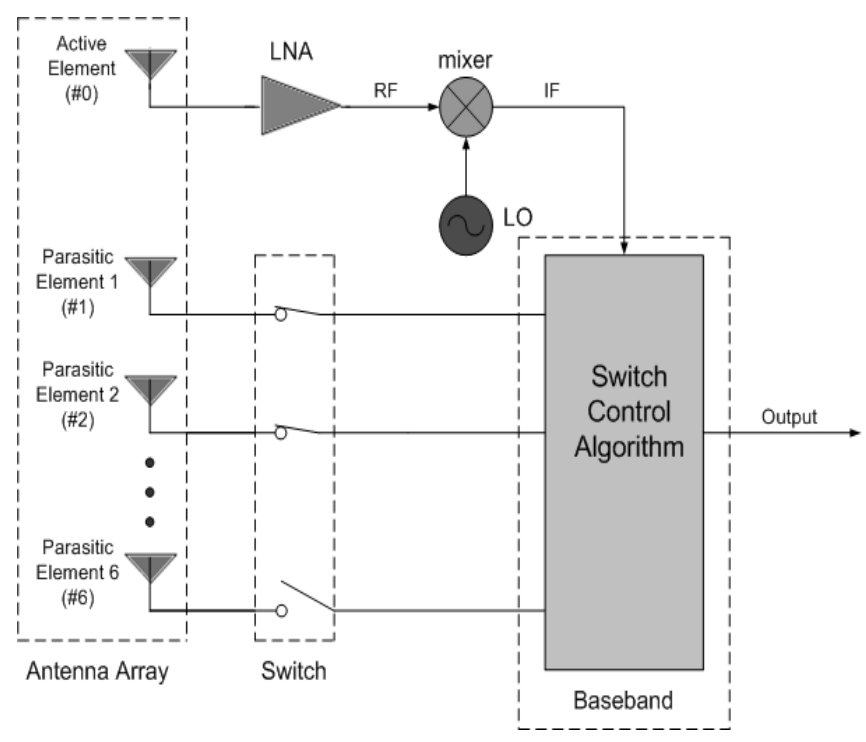

(b)

Fig.3 (a) a simplistic example of beam-forming circuit with parasitic elements controlled by switches for PASHE and PASCE; (b) a typical aerial beam-forming structure with parasitic elements using switch control for PASHE and PASCE 


\section{Simulation AND MeAsurement Results}

Analytical formulation of the coupling generated by each patch antenna element would be a formidable task, thus such numerical modeling using the finite element method (FEM) was implemented by HFSS [6] instead. HFSS is a 3D simulation software for calculating the electromagnetic behavior of a structure. It has higher simulation accuracy compared to other simulation software tools mainly due to the assumption of the ground plane to be finite rather than infinite, thus the simulation environment has more similarity to reality. To obtain the optimal configuration, both antennas were optimized with the steepest gradient optimization procedure.

Simulations were performed for both patch antennas with one parasitic element open-circuited and remaining shortcircuited to ground at six directions in azimuth plane with identical angle difference which is $60^{\circ}$. Both simulated and measured reflection coefficients as a function of frequency were obtained and compared in Fig.4 (a) and (b) for PASHE and PASCE respectively when the main beam lobe directs to a specific orientation in azimuth plane. It can be seen from Fig.4 (a) and (b), the curve of the reflection coefficient from measurement has similar pattern to that from simulation for both PASHE and PASCE. However, the curve of the reflection coefficient from measurement slightly shifts to the left of that from simulation for both PASHE and PASCE. The frequency shift is possibly due to the error of the dielectric constant in FR-4 epoxy/fiber-glass at $2.4 \mathrm{GHz}$ and antenna fabrication. The resonant frequency, reflection coefficient and $10 \mathrm{~dB}$ bandwidth of PASHE and PASCE are compared in TABLE I. It is seen from TABLE I, PASHE has larger bandwidth which is $5.97 \%$ to the measured resonant frequency compared to PASCE of which the bandwidth is only $3.53 \%$ to the measured resonant frequency. The increas-

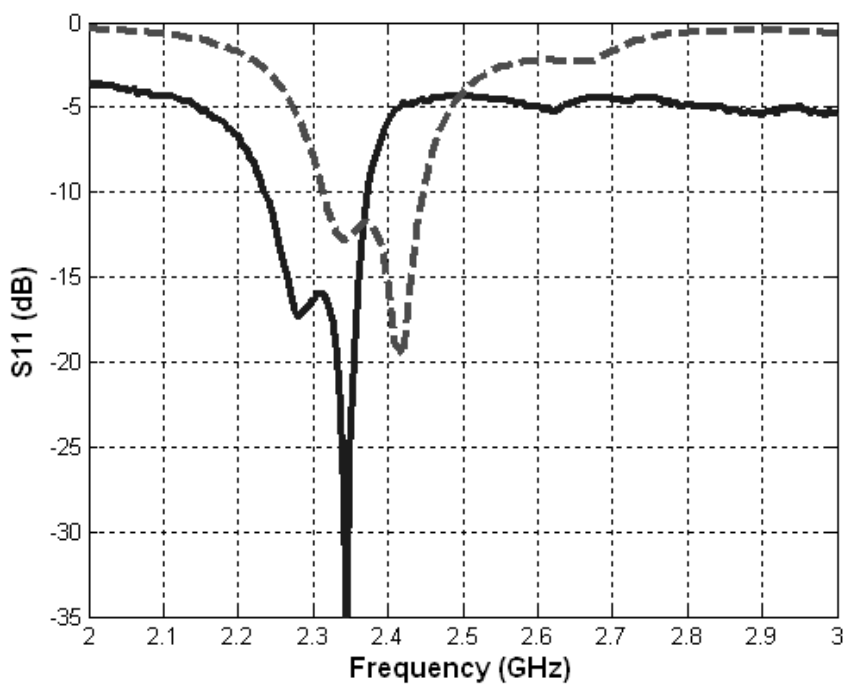

- Measured $\mathrm{S}_{11}$ of PASHE

(a)

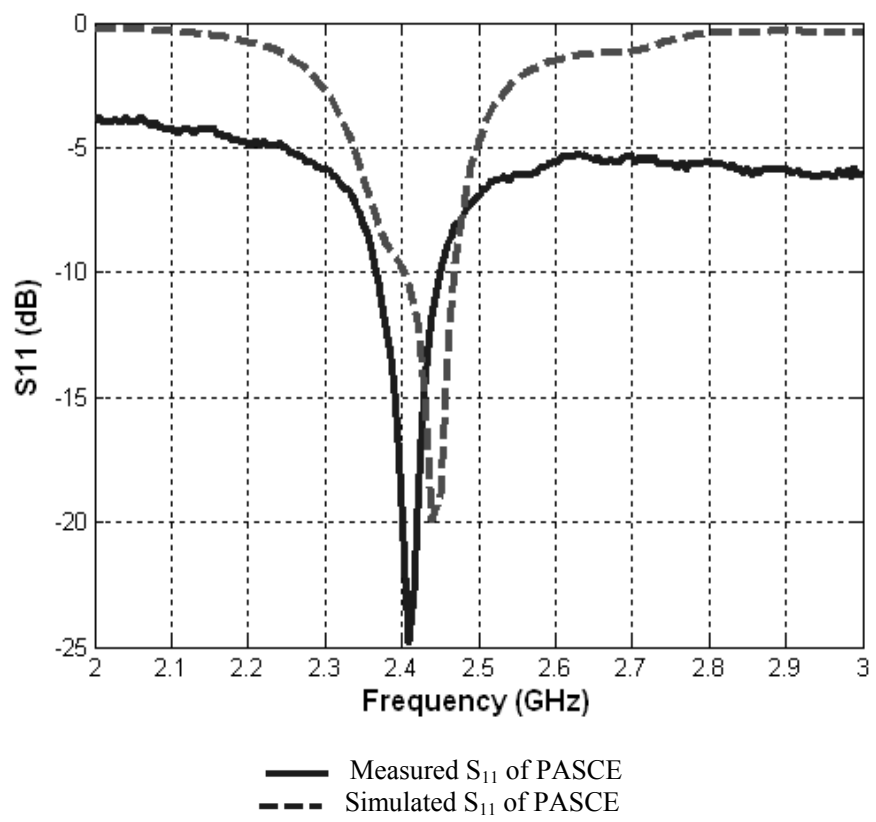

(b)

Fig.4 Simulated and measured reflection coefficients of PASHE and PASCE when the parasitic element No. 6 is open-circuited while remaining shortcircuited to ground: (a) PASHE; (b) PASCE

TABLE I

Comparison of Measured and Simulated Resonant Frequency, Reflection Coefficient and BANDwidTh For Pashe AND Pasce

\begin{tabular}{|c|c|c|c|c|}
\hline & \multicolumn{2}{|c|}{ PASHE } & \multicolumn{2}{c|}{ PASCE } \\
\cline { 2 - 5 } \multicolumn{2}{|c|}{ Reasurement } & Simulation & Measurement & Simulation \\
\hline $\begin{array}{c}\text { Resonant } \\
\text { Frequency } \\
(\mathrm{GHz})\end{array}$ & 2.345 & 2.420 & 2.410 & 2.440 \\
\hline $\begin{array}{c}\text { Reflection } \\
\text { Coefficient } \\
(\mathrm{dB})\end{array}$ & -35.00 & -19.64 & -24.80 & -19.90 \\
\hline $\begin{array}{c}\text { 10dB } \\
\text { Bandwidth } \\
(\mathrm{MHz})\end{array}$ & 140 & 140 & 85 & 70 \\
\hline
\end{tabular}

ed bandwidth produced by PASHE is possibly attributed to the increased mutual coupling of the hexagonal shaped elements.

The simulated radiation patterns in the elevation plane in far field with left-hand circular polarization of PASHE and PASCE are illustrated in Fig.5 (a) and (b) respectively while the main beam lobe is steered in different directions in azimuth plane. It is seen from Fig.5 (a) and (b), the simulated radiation pattern of PASHE in elevation plane has slightly difference from that of PASCE when the main beam lobe of each antenna is steered to a specific direction in azimuth plane. Furthermore, the radiation pattern of both antennas in elevation plane is not exactly identical for each of the six directions in azimuth plane. This possibly results from the imperfect left-hand circular polarization. The characteristics of the radiation pattern in elevation plane such as elevation angle, maximum gain and $3 \mathrm{~dB}$ beam-width are compared for PASHE and PASCE respectively in TABLE II. TABLE II 
reveals that PASCE has slightly larger elevation angle, maximum gain and $3 \mathrm{~dB}$ beam-width compared to PASHE.

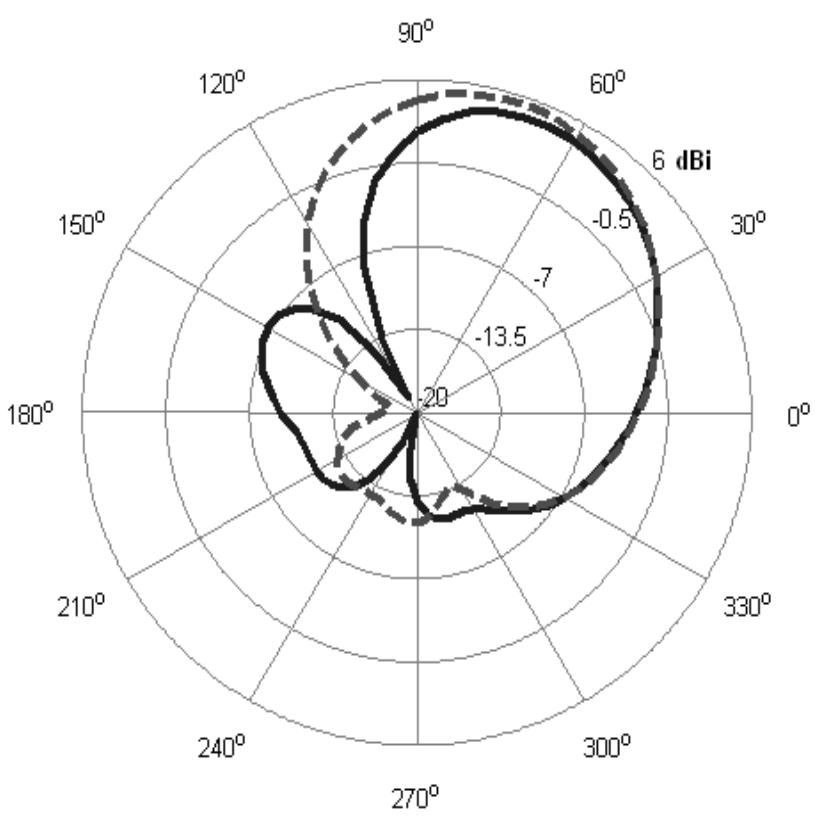

Radiation pattern of PASHE in elevation plane - - Radiation pattern of PASCE in elevation plane

(a)

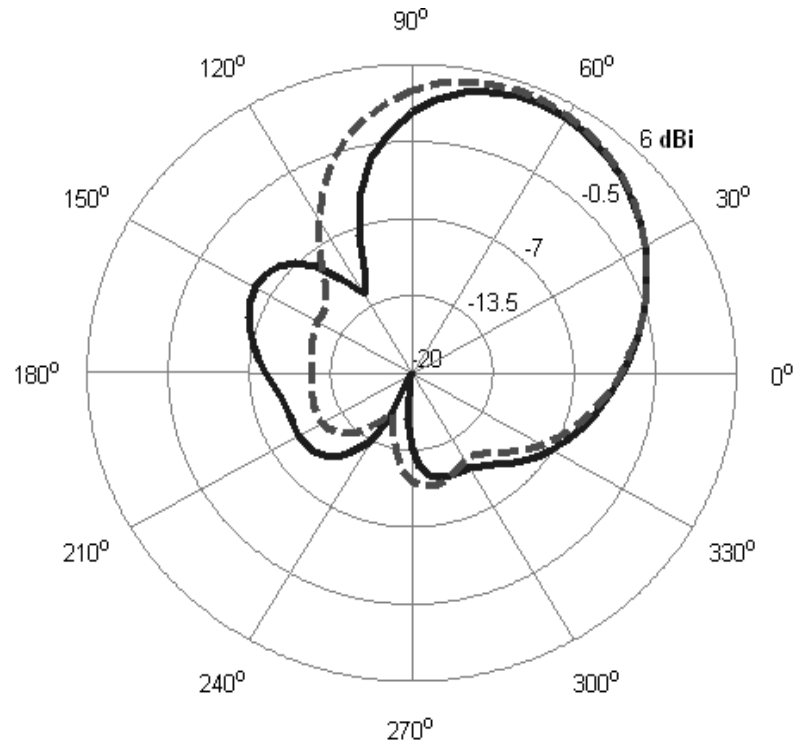

Radiation pattern of PASHE in elevation plane Radiation pattern of PASCE in elevation plane

(b)

Fig.5 Simulated radiation pattern of PASHE and PASCE in elevation plane when the main beam lobe is steered in different directions in azimuth plane:

(a) the parasitic element No.6 is open-circuited while remaining short-

circuited to ground; (b) the parasitic element No.3 is open-circuited while remaining short-circuited to ground.
TABLE II

Comparison of the Characteristics of Simulated Radiation Pattern in Elfyation Plane in two Different Directions in Azimuth Plane for PASHE AND PASCE

\begin{tabular}{|c|c|c|c|c|}
\hline & \multicolumn{2}{|c|}{ PASHE } & \multicolumn{2}{c|}{ PASCE } \\
\cline { 2 - 5 } & $\begin{array}{c}\text { Case in } \\
\text { Fig.5 (a) }\end{array}$ & $\begin{array}{c}\text { Case in } \\
\text { Fig.5 (b) }\end{array}$ & $\begin{array}{c}\text { Case in } \\
\text { Fig.5 (a) }\end{array}$ & $\begin{array}{c}\text { Case in } \\
\text { Fig.5 (b) }\end{array}$ \\
\hline $\begin{array}{c}\text { Elevation Angle } \\
\text { (deg) }\end{array}$ & $65^{\circ}$ & $65^{\circ}$ & $70^{\circ}$ & $70^{\circ}$ \\
\hline $\begin{array}{c}\text { Maximum Gain } \\
(\mathrm{dBi})\end{array}$ & 4.64 & 4.90 & 5.44 & 5.27 \\
\hline $\begin{array}{c}3 \mathrm{~dB} \text { Beam-width } \\
(\mathrm{deg})\end{array}$ & $60^{\circ}$ & $60^{\circ}$ & $65^{\circ}$ & $65^{\circ}$ \\
\hline
\end{tabular}

\section{CONCLUSION}

Two novel smart patch antenna arrays, namely PASHE and PASCE with beam-switching function were discussed in this paper. The performances of both antenna arrays were compared under the same simulation and testing environment. The result of reflection coefficient as a function of frequency obtained from both simulation and measurement reveals PASHE have a larger bandwidth compared to PASCE due to the better edge effect of mutual coupling. Therefore the hexagonal shape can be applied for designing microstrip patch element to effectively increase the bandwidth thus overcome the inherent defect of narrow bandwidth of microstrip patch antenna. Future work is being conducted to generate the circular polarization for PASHE and PASCE by a single active port utilizing slot perturbation for the ease of testing and economical consideration. These smart patch antennas with high performances, low profile, and simplistic beam-controlling circuit are becoming potential prototypes to meet the demand for increasing indoor mobile wireless terminals.

\section{REFERENCES}

[1] R. Harrington, "Reactively controlled directive arrays," IEEE Transactions on Antennas and Propagation, vol. ap-26, no. 3, pp. 390395, May 1978.

[2] R. Schlub, J. Lu and T. Ohira, J. Lu, and T. Ohira, "Seven element ground skirt monopole ESPAR antenna design from a genetic algorithm and the finite element method," IEEE Transactions on Antennas and Propagation, vol. 51, no. 11, pp. 3033-3039, Nov. 2003.

[3] J. Lu, D. Ireland, and R. Schlub, "Dielectric embedded ESPAR (DEESPAR) antenna array for wireless communications," IEEE Transactions on Antennas and Propagation, vol. 53, no. 8, pp. 2437 2443, Aug. 2005.

[4] D. Gray, J. Lu, and D. Thiel, "Electronically steerable Yagi-Uda microstrip patch antenna array," IEEE Transactions on Antennas and Propagation, vol. 46, no. 5, pp. 605-608, May 1998.

[5] D. Thiel and S. Smith, Switched Parasitic Antennas for Cellular Communications, Norwood, MA, Artech House, Inc., 2001.

[6] HFSS v9.0. (2004). Ansoft Corporation. [Online]. Available: http://www.ansoft.com/products/hf/hfss/ 